\title{
The European Network For Understanding Mechanisms Of Severe Asthma study
}

\section{To the Editor:}

We found the results of the European Network For Understanding Mechanisms Of Severe Asthma (ENFUMOSA) study [1] and its accompanying Editorial [2] very interesting and would like to raise two issues, one regarding classification of subjects, and the other concerning pathogenesis. The ENFUMOSA study defined a group with severe asthma, requiring high-dose inhaled corticosteroids who continued to experience severe exacerbations of asthma. These subjects differed from the comparison group by having less atopy and increased sputum neutrophils. It was hypothesised that other environmental factors, such as infections, may be important.

We recently reviewed the inflammatory phenotype and mechanisms of asthma and drew attention to a phenotype of noneosinophilic asthma [3]. These subjects had symptomatic asthma and airway hyperresponsiveness but normal levels of sputum eosinophils. Noneosinophilic asthma was observed to occur in all grades of asthma severity, including steroid-naïve mild asthma [3]. In persistent asthma, the noneosinophilic asthma phenotype was characterised by increased levels of sputum neutrophils and increased interleukin (IL)-8 [4]. Thus, there is a strong alignment between the results of the ENFUMOSA study and our work describing noneosinophilic asthma. Rather than being restricted to severe asthma, as the ENFUMOSA study suggests, this inflammatory phenotype occurs across the severity spectrum of persistent asthma. This suggests that neutrophilic inflammation causing noneosinophilic asthma may by a specific class of asthma, rather than a stage in the progression of asthma. Clarification of this important point will require longitudinal studies.

It would be useful to re-examine the data from the ENFUMOSA study, classified by inflammatory phenotype. There are now reliable data on the normal levels of inflammatory cells in induced sputum [4] and given the reported increased neutrophils in the subjects with severe asthma, this classification may provide important additional information about noneosinophilic asthma. As suggested in the Editorial [2], previous work provided important evidence that severe asthma can be classified based upon inflammatory phenotype into eosinophilic and noneosinophilic severe asthma. This classification provided the first suggestion that severe asthma may involve different inflammatory phenotypes and processes with similar resultant clinical presentation.

The potential mechanisms of noneosinophilic asthma are interesting. We hypothesised that noneosinophilic asthma may represent persistent activation of the innate immune response [3]. Innate immune activation leads to activation of a common transcriptional programme involving nuclear factor $\kappa \mathrm{B}$, IL-8, and consequent neutrophilic inflammation. Infectious agents are key players in the activation of this arm of the immune system. Based on these observations, we would interpret the findings of the ENFUMOSA study as being consistent with infectious agents triggering innate immune activation to explain the clinical observations and inflammatory changes observed in severe asthma. This would also explain why exacerbations continued to occur despite the effects of steroids and their potent antieosinophilic action.
If these hypotheses that noneosinophilic asthma represents a different class of asthma triggered by innate immune activation are correct, then this has important implications for the choice of therapy in asthma.

\section{P.G. Gibson*, J.L. Simpson ${ }^{\#}$}

*Dept of Respiratory and Sleep Medicine, University of Newcastle, and "John Hunter Hospital, Hunter Medical Research Institute, Newcastle, New South Wales, Australia.

\section{References}

1. Holgate ST. The ENFUMOSA cross-sectional European multicentre study of the clinical phenotype of chronic severe asthma. Eur Respir J 2003; 22: 470-477.

2. Wenzel S. A different disease, many diseases or mild asthma gone bad? Challenges of severe asthma. Eur Respir J 2003; 22: 397-398.

3. Douwes J, Gibson P, Pekkanen J, Pearce N. Non-eosinophilic asthma: importance and possible mechanisms. Thorax 2002; 57: 643-648.

4. Gibson PG, Simpson JL, Saltos N. Heterogeneity of airway inflammation in persistent asthma: evidence of neutrophilic inflammation and increased sputum interleukin-8. Chest 2001; 119: 1329-1336.

\section{From the authors:}

We thank P.G. Gibson and J.L. Simpson for their interest and kind comments on the report of the cross-sectional European Network For Understanding Mechanisms Of Severe Asthma (ENFUMOSA) study. We also believe that asthma is a syndrome where different pathogenetic mechanisms are involved in different groups of patients. With regard to airway neutrophilia and asthma subtypes, elite skiers in the Nordic countries often acquire airway hyperresponsiveness and a variable airflow obstruction that may meet clinical criteria for the diagnosis of asthma [1]. The numbers of neutrophils in the bronchial biopsies were twice as high among elite skiers as in a group of subjects with mild asthma, and there was also evidence of airway remodelling as in asthma [2]. However, the histopathology in the elite athletes also displayed dissimilarities, such as bronchus-associated lymphoid tissue aggregates [3]. As also discussed by P.G. Gibson and J.L. Simpson, inappropriate activation of the innate immune response could be one potential mechanism involved in the development of airway hyperresponsiveness and obstruction associated with neutrophilia.

P.G. Gibson and J.L. Simpson raise the possibility that the neutrophilic phenotype we observed among subjects with severe asthma in the ENFUMOSA study was a reflection of one asthma phenotype that is expressed across the spectrum of disease severities, rather than being specifically associated 
with severe asthma. Our findings would at first glance seem to speak against this proposal. Thus, there was a significant accumulation of subjects with neutrophilic inflammation amongst the patients with severe asthma in the ENFUMOSA study and this was not seen in the group with mild-tomoderate controlled persistent asthma investigated with the same procedures. However, as discussed in the Editorial [4] accompanying our paper [5], there are subgroups of subjects with severe asthma that have eosinophilic inflammation. As it is established that mild-to-moderate allergic asthma is most commonly associated with an eosinophilic inflammation, our findings could lead to a hypothesis that eosinophilic asthma provides relative protection against progression into severe disease. This would also fit with the observation that atopy was less common among the subjects with severe asthma, as documented in the ENFUMOSA study.

Rather than speculating further, or engage in the debate on whether the eosinophil is pro- or anti-inflammatory, or both, the ENFUMOSA group has already embarked on the longitudinal study that P.G. Gibson and J.L. Simpson suggest. We are in the middle of the Longitudinal Assessment of Clinical Course and BIOmarkers in Severe Chronic AIRway Disease (BIOAIR) study, a large Pan-European and partly European Union sponsored effort, where genotypes, biomarkers and clinical characteristics are followed in three groups of patients with mild asthma, severe asthma and chronic obstructive pulmonary disease. Following an intensive cross-sectional baseline characterisation of the subjects, they are monitored with repeated measures of biomarkers, airway function and a large panel of other investigations over at least $1 \mathrm{yr}$. We hope that these data may help clarify some of the hypotheses that arose from the ENFUMOSA study. The BIOAIR study will be completed in 2005 and we hope to be able to report on the findings soon after. We find it particularly important in the BIOAIR study to have repeated measurements of the different variables across 12 months, as many hypotheses concerning pathogenesis are based on cross-sectional observations in relatively small study populations, rather than demonstrating consistent differences that are preserved over time. We hope that the results of the BIOAIR study, which also includes a double-blind placebo-controlled oral steroid intervention, may provide data to help us respond more comprehensively to P.G. Gibson's and J.L. Simpson's question concerning the classification and pathogenesis of asthma.
We would finally like to broaden the view beyond the debate of eosinophilic and noneosinophilic inflammation. The different asthma phenotypes are the result of complex geneenvironment interactions where we currently, despite exciting recent progress, only see the tip of the iceberg. It is likely that small differences in genotype may determine how common environmental influences produce varying phenotypes. Along these lines, it was recently observed that a specific and functionally important polymorphism of the cell surface receptor used by hepatitis A virus may protect individuals from development of atopy after hepatitis infection [6]. Many similar observations are likely to follow and may in the future help us to identify the genetic and environmental causes of distinct expressions of the heterogenous syndrome of asthma.

\section{S.T. Holgate*, S-E. Dahlén ${ }^{\#}$}

*University of Southampton, Southampton, UK. ${ }^{\#}$ Karolinska Institutet, Stockholm, Sweden.

\section{References}

1. Larsson K, Ohlsen P, Larsson L, Malmberg P, Rydström PO, Ulriksen $\mathrm{H}$. High prevalence of asthma in cross country skiers. BMJ 1993; 307: 1326-1329.

2. Karjalainen EM, Laitinen A, Sue-Chu M, Altraja A, Bjermer L, Laitinen LA. Evidence of airway inflammation and remodelling in ski athletes with and without bronchial hyperresponsiveness to methacholine. Am J Respir Crit Care Med 2000; 161: 2086-2091.

3. Sue-Chu M, Karjalainen EM, Altraja A, et al. Lymphoid aggregares in endobronchial biopsies from young elite crosscountry skiers. Am J Respir Crit Care Med 1998; 158: 597601.

4. Wenzel S. A different disease, many diseases or mild asthma gone bad? Challenges of severe asthma. Eur Respir J 2003; 22: $397-398$

5. Holgate ST. The ENFUMOSA cross-sectional European multicentre study of the clinical phenotype of chronic severe asthma. Eur Respir J 2003; 22: 470-477.

6. McIntire JJ, Umetsu SE, Macaubas C, et al. Immunology: hepatitis A virus link to atopic disease. Nature 2003; 425 576.

\section{Host response to transmissible Pseudomonas aeruginosa}

\section{To the Editor:}

The recent study by JoNES et al. [1] was interesting in that it found no significant difference in local airway or systemic inflammation between cystic fibrosis patients colonised with either transmissible or nontransmissible strains of Pseudomonas aeruginosa. At face value these results appear reassuring, although the authors do rightly raise a note of caution. One factor that was not considered in this article is the effect of bacterial load within the airways, but this must surely be the denominator when one is trying to identify a significant difference in host response. There is also anecdotal evidence that the host inflammatory response at the time of first acquisition of transmissible $P$. aeruginosa or Burkholderia cepacia may be what determines subsequent outcome i.e. some patients become extremely unwell and die whereas the majority seem to remain relatively well. Presumably some sort of status quo is reached between host and organism and perhaps what we are seeing in the study by JONES et al. [1] is in fact a survivor effect. The experience of ARMSTRONG et al. [2], where a number of deaths occurred shortly after acquisition of a transmissible strain goes some way toward supporting this hypothesis. However, the long-term outcome in those other children in the study by ARMSTRONG et al. [2] who were similarly infected remains unknown, but hopefully longitudinal follow-up in these individuals will add further weight to the reassurance by JONES et al. [1].

In the meantime, until there is evidence to the contrary, 